ARTICLE

Received 25 Jul 2013 | Accepted 14 Nov 2013 | Published 12 Dec 2013 DOI: 10.1038/ncomms3933

\title{
Co-operativity in a nanocrystalline solid-state transition
}

Sarah L. White ${ }^{1}$, Jeremy G. Smith¹, Mayank Behl ${ }^{2} \&$ Prashant K. Jain 1,3,4

Co-operativity is a remarkable phenomenon mostly seen in biology, where initial reaction events significantly alter the propensity of subsequent reaction events, giving rise to a nonlinear tightly regulated synergistic response. Here we have found unique evidence of atomic level co-operativity in an inorganic material. A thousand-atom nanocrystal (NC) of the inorganic solid cadmium selenide exhibits strong positive co-operativity in its reaction with copper ions. A NC doped with a few copper impurities becomes highly prone to be doped even further, driving an abrupt transition of the entire NC to the copper selenide phase, as manifested by a strongly sigmoidal response in optical spectroscopy and electron diffraction measurements. The examples presented here suggest that cooperative phenomena may have an important role in the solid state, especially in the nucleation of new chemical phases, crystal growth, and other materials' transformations.

\footnotetext{
${ }^{1}$ Department of Chemistry, University of Illinois at Urbana Champaign, 600 South Mathews Avenue, Urbana, Illinois 61801, USA. ${ }^{2}$ Department of Chemical and Biomolecular Engineering, University of Illinois at Urbana Champaign, 600 South Mathews Avenue, Urbana, Illinois 61801, USA. ${ }^{3}$ Materials Research Lab, University of Illinois at Urbana Champaign, 104 South Goodwin Avenue, Urbana, Illinois 61801, USA. ${ }^{4}$ Beckman Institute for Advanced Science and Technology, University of Illinois at Urbana Champaign, 405 North Mathews Avenue, Urbana, Illinois 61801, USA. Correspondence and requests for materials should be addressed to P.K.J. (email: jain@illinois.edu).
} 
S ince the first observation in 1910 of the co-operative binding of oxygen to haemoglobin ${ }^{1,2}$, where binding at any of the four sites of haemoglobin increases the oxygen affinity of the other sites, co-operative phenomena have been displayed in a host of macromolecular systems ${ }^{3}$, ranging from the unwinding of DNA and protein folding ${ }^{4}$ to self-assembly in supramolecular systems ${ }^{5}$. A common feature of co-operative systems is that they possess multiple binding sites with some mechanism for feedback or coupling between the binding sites. The feedback itself may be positive or negative. Positive cooperativity is manifested as an apparent all-or-nothing behaviour $^{6}$ : rather than making a gradual progression through intermediary states, the system undergoes a relatively abrupt transition from the initial to the final state in response to an extremely small change in conditions (for example, ligand, denaturing agent, $\mathrm{pH}$ or temperature). The system spends little time in the intermediary states, resulting in their low population. The strongly sigmoidal oxygen-binding curve of haemoglobin is the archetypal example of such behaviour ${ }^{1}$.

Although examples of co-operativity seem limited to macromolecules, mostly biological ${ }^{3}$, we found evidence of co-operative behaviour in an inorganic solid material. The observation was made in nanocrystals $(\mathrm{NCs})$ of cadmium selenide $(\mathrm{CdSe})^{7}$ undergoing a cation exchange in the presence of monovalent ions as:

$$
(\mathrm{CdSe})_{n}+2 n \cdot \mathrm{Cu}_{(\mathrm{aq})}^{+} \rightleftharpoons\left(\mathrm{Cu}_{2} \mathrm{Se}\right)_{n}+\mathrm{n} \cdot \mathrm{Cd}_{(\mathrm{aq})}^{2+} .
$$

In this transformation ${ }^{8,9}$, the $\mathrm{Cd}^{2+}$ cationic sub-lattice is replaced with $\mathrm{Cu}^{+}$ions, two for every $\mathrm{Cd}^{2+}$ ion, to form a $\mathrm{NC}$ of cuprous selenide $\left(\mathrm{Cu}_{2} \mathrm{Se}\right)$. Each CdSe $\mathrm{NC}$ is $\sim 4 \mathrm{~nm}$ or $\sim 1,300$ atoms in size, and loosely speaking, can be thought of as a 'macromolecular system' with multiple 'sites' for foreign cations. The cation exchange reaction ${ }^{10}$ itself has received a lot of fundamental attention because of its remarkably fast (millisecond timescale) kinetics ${ }^{11}$ in nanosized crystals. The transformation, if well understood, can be exploited for topotaxially templated synthesis of nanostructures with novel compositions and morphologies ${ }^{12,13}$, designing heterojunctions in $\mathrm{NCs}^{14}$, sensing heavy metal ions ${ }^{15}$, and achieving electronic doping ${ }^{16,17}$.

With limited mechanistic insight available, cation exchange is often assumed to be a diffusion-limited transformation: as long as the cation can diffuse to a lattice site, exchange can take place at that site. By this assumption, all lattice sites exposed to $\mathrm{Cu}^{+}$ would have an equal affinity for exchange and the CdSe ensemble would transform in a random site-by-site manner to the $\mathrm{Cu}_{2} \mathrm{Se}$ phase as adequate $\mathrm{Cu}^{+}$becomes available. However, our optical and electron diffraction measurements show that the exchange transformation does not follow such a memoryless, diffusionlimited process. Instead, we see positive co-operative behaviour, where initial $\mathrm{Cu}^{+}$doping of a NC strongly enhances the likelihood of further $\mathrm{Cu}^{+}$doping within the same NC, causing the NC to transition sharply from a lightly doped CdSe NC to the $\mathrm{Cu}_{2} \mathrm{Se}$ phase.

\section{Results}

Optical probing of cation exchange. We used excitonic absorption spectra (Fig. 1a) to monitor the cation exchange of a solution of 4-nm CdSe NCs as it was titrated with $\mathrm{Cu}^{+}$. With increasing $\mathrm{Cu}^{+}$feed concentration, the excitonic band at $2.1 \mathrm{eV}$, corresponding to the $1 \mathrm{~S}_{\mathrm{h}}-1 \mathrm{~S}_{\mathrm{e}}$ transition in the CdSe NCs, decreased in intensity, which is expected as the conversion to $\mathrm{Cu}_{2} \mathrm{Se}$ proceeded. Concomitantly, the absorption in the nearinfrared region associated with $\mathrm{Cu}_{2} \mathrm{Se}$ increased.

Two important features emerge in these spectra. First, the spectral position of the CdSe exciton stayed relatively unchanged throughout the titration, which indicates an absence ${ }^{13}$ of any significant fraction of binary $\mathrm{CdSe} / \mathrm{Cu}_{2} \mathrm{Se} \mathrm{NCs}$ formed by partial cation exchange. Such heterostructures would exhibit strongly shifted excitonic peaks, because the excitonic peak position is highly dependent on the CdSe crystallite size ${ }^{18}$. Although it is possible that partial conversion of a $\mathrm{CdSe} \mathrm{NC}$ to $\mathrm{Cu}_{2} \mathrm{Se}$ completely quenches its excitonic absorption, this possibility is excluded by a key observation: the decrease in the fraction of CdSe NCs (estimated from the CdSe exciton height) is perfectly correlated with the increase in the fraction of $\mathrm{Cu}_{2} \mathrm{Se} \mathrm{NCs}$ (estimated from the $\mathrm{Cu}_{2} \mathrm{Se}$ absorbance at $1.82 \mathrm{eV}$ ), as shown in Fig. 2. A clear isosbestic point (Fig. 1a) is identified, which is a well-known indicator of a two species system, where one species converts to another over the course of the titration. Thus, it appears that individual CdSe NCs switch fully to $\mathrm{Cu}_{2} \mathrm{Se}$ as sufficient $\mathrm{Cu}^{+}$becomes available. With increasing amount of $\mathrm{Cu}^{+}$, more and more NCs make this transition from CdSe to $\mathrm{Cu}_{2} \mathrm{Se}$. Thus, each NC is either in the CdSe state (albeit with some $\mathrm{Cu}^{+}$dopants) or in the fully exchanged $\mathrm{Cu}_{2} \mathrm{Se}$ state, manifesting the classic two-state behaviour of a co-operative system.

Across a range of systems, sigmoidal-binding curves are a definitive manifestation of co-operative behaviour ${ }^{1}$. Figure 2 shows such a curve for the cation exchange process: this plot of the fraction of NCs in the CdSe state as a function of $\mathrm{Cu}^{+}$ concentration is strikingly sigmoidal, as also is the plot of the fraction of $\mathrm{NCs}$ in the $\mathrm{Cu}_{2} \mathrm{Se}$ state. Only a small to negligible fraction of NCs is exchanged at low $\mathrm{Cu}^{+}$feed concentrations. However, once a critical concentration is reached, a major fraction of $\mathrm{NCs}$ switch to the $\mathrm{Cu}_{2} \mathrm{Se}$ state over a narrow concentration window about the critical concentration. The titration curve fits well $(R=0.996)$ the Hill equation ${ }^{1}$, with a Hill coefficient or co-operativity factor of $n_{\mathrm{H}}=7.1$. As a comparison, strongly co-operative oxygen binding by haemoglobin shows a value of $n_{\mathrm{H}}=2.3-3.0$. We have found some experiment-toexperiment variation in the measured $n_{\mathrm{H}}$ (range 4-7). Size/shape/ surface-related heterogeneities inherent to NC samples may contribute to such variation.

Structural probing of cation exchange. Electron diffraction measurements (Fig. 3) provide structural evidence of the sharp onset of the cation exchange process. The initial NC samples show wurtzite CdSe diffraction features, which persist up to a $\mathrm{Cu}^{+}$feed concentration as high as 549 ions per NC (Table 1). Around this $\mathrm{Cu}^{+}$concentration (the half-way point), a significant fraction of NCs undergo a conversion to $\mathrm{Cu}_{2} \mathrm{Se}$, as indicated by the emergence of diffraction features corresponding to the tetragonal belladoite phase of $\mathrm{Cu}_{2} \mathrm{Se}$, albeit superimposed on wurtzite diffraction features from the remnant CdSe NCs. With further addition of $\mathrm{Cu}^{+}$, the tetragonal $\mathrm{Cu}_{2} \mathrm{Se}$ diffraction pattern becomes more prominent as additional NCs convert to $\mathrm{Cu}_{2} \mathrm{Se}$. Concomitantly, the wurtzite $\mathrm{CdSe}$ diffraction pattern wanes. Eventually, at about $1,317 \mathrm{Cu}^{+}$ions per NC (slightly higher than a full stoichiometric amount), only the tetragonal $\mathrm{Cu}_{2} \mathrm{Se}$ diffraction pattern is seen, indicating conversion of all NCs to $\mathrm{Cu}_{2} \mathrm{Se}$.

$\mathrm{Cu}^{+}$doping as a precursor to exchange. In the regime below the critical concentration, it is clear that there is little conversion to $\mathrm{Cu}_{2} \mathrm{Se}$ and most of the NCs exhibit the CdSe crystal structure. However, in this regime, the NCs are doped with $\mathrm{Cu}^{(1+)}$ impurities, as found from X-ray photoelectron spectroscopy (XPS) (Fig. 4b,c and Supplementary Fig. S1) and elemental analyses (Supplementary Fig. S2 and Supplementary Table S1). $\mathrm{Cu}^{+}$ is a common interstitial dopant in $\mathrm{CdSe}^{19,20}$. It is known that such dopants do not necessarily disrupt the excitonic band or the 
a

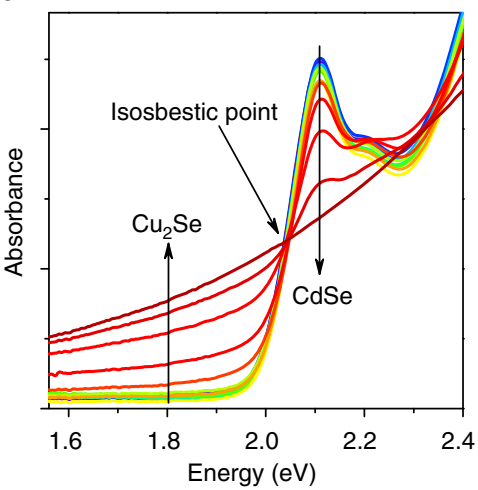

b

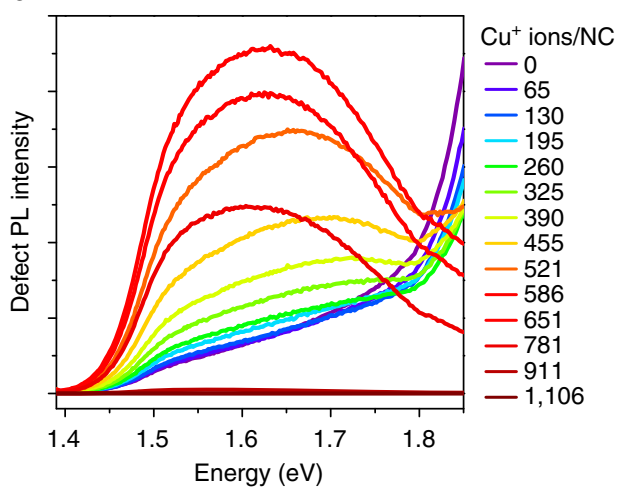

Figure 1 | Optical monitoring of the cation exchange reaction of CdSe $\mathbf{N C s}$ with $\mathbf{C u}^{+}$. Excitonic absorption spectra (a) of an ensemble of $\mathrm{CdSe}$ NCs as a function of added $\mathrm{Cu}^{+}$. Feed $\mathrm{Cu}^{+}$concentrations are denoted as the number of ions available per $\mathrm{CdSe}$ NC. With increasing addition of $\mathrm{Cu}^{+}$, the $\mathrm{CdSe} \mathrm{NC}$ exciton peak at $2.1 \mathrm{eV}$ decreases without undergoing any shift. Concomitantly, the $\mathrm{Cu}_{2} \mathrm{Se}$ absorption in the near-infrared increases. A clear isosbestic point is seen. Following complete exchange, the absorption spectrum of the NCs resembles that of $\mathrm{Cu}_{2} \mathrm{Se}$ with a bandgap onset around $1 \mathrm{eV}$. Note, $\mathrm{Cu}_{2} \mathrm{Se}$ is an indirect semiconductor and therefore has no excitonic band, rather only a featureless absorption above its bandgap. PL spectra (b) in the sub-bandgap region show the emergence of defect luminescence when $\mathrm{Cu}^{+}$is added, which evolves further with increasing $\mathrm{Cu}^{+}$feed concentration. The excitonic bandgap PL of CdSe NCs was subtracted out from these spectra, as detailed in the Methods section.

a

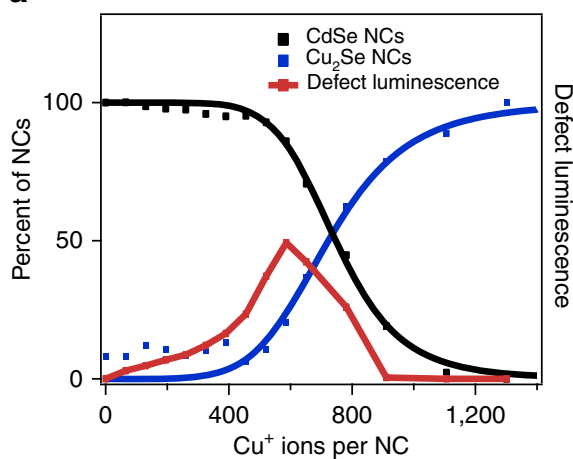

b

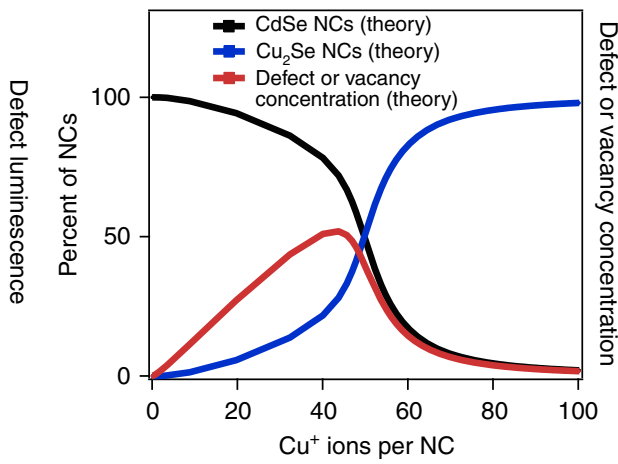

Figure 2 | Sigmoidal nature of the cation exchange titration. Experimental plot (a) of the fraction of NCs in the CdSe state (black) and those in the $\mathrm{Cu}_{2} \mathrm{Se}$ state (blue) as a function of added $\mathrm{Cu}^{+}$is strikingly sigmoidal, representative of a strongly co-operative process. Below a critical concentration of $\sim 500 \mathrm{Cu}^{+}$ions per NC, very few NCs undergo a transition to the $\mathrm{Cu}_{2} \mathrm{Se}$ state. A fit to the Hill plot (solid black line, $R=0.996$ ) yields a co-operativity factor of $n_{\mathrm{H}}=7$.1. Note we use total $\mathrm{Cu}^{+}$concentration, although the Hill equation is strictly valid for free concentrations. The fraction of NCs in the $\mathrm{CdSe}$ state was determined from the height of the $\mathrm{CdSe}$ excitonic peak from Fig. 1a and the fraction in the $\mathrm{Cu}_{2} \mathrm{Se}$ state was determined from the $\mathrm{Cu}_{2} \mathrm{Se}$ band-to-band absorption at $1.82 \mathrm{eV}$. The procedure for analysis is described in Methods. The rise in the $\mathrm{Cu}_{2} \mathrm{Se}$ fraction tracks well with the drop in the $\mathrm{CdSe}$ fraction, further confirming two-state behaviour. The defect luminescence intensity (red), integrated across the broad band shown in Fig. $1 \mathrm{~b}$ increased as $\mathrm{Cu}^{+}$was added, until it reached a maximum around the critical concentration, beyond which it decreased, until it reached zero. A theoretical simulation (b) of a 20-site NC reproduced the observed nature of the cation exchange transformation. Sequential binding was used to model the positive co-operative behaviour. The simulated fraction of $\mathrm{Cu}_{2} \mathrm{Se} \mathrm{NCs}$ in solution as a function of the total $\mathrm{Cu}^{+}$concentration, plotted as the number of ions per $\mathrm{NC}$, follows a strongly sigmoidal curve (blue). The fraction of all NCs in the CdSe state, either pure or doped, is also shown (black). The simulated concentration of $\mathrm{Cu}^{+}$dopants and associated $\mathrm{Cd}^{2}+$ vacancies (red) reproduces the observed evolution of the defect luminescence. The defect concentration reaches a maximum around the critical $\mathrm{Cu}^{+}$concentration, beyond which it gradually decreases to zero.

overall crystallographic structure of $\mathrm{CdS} \mathrm{e}^{18,19}$, which is consistent with our optical and electron diffraction measurements.

$\mathrm{Cu}^{+}$doping can, however, cause lattice defects: for instance, for every two interstitial $\mathrm{Cu}^{+}$impurities in a $\mathrm{NC}$, charge compensation would require the formation of a $\mathrm{Cd}^{2}+$ vacancy. Both $\mathrm{Cu}^{+}$impurities and vacancies serve as mid-gap trap states and give rise to sub-bandgap luminescence ${ }^{21-24}$, which we employed for monitoring the doping of NCs at various stages in the cation exchange titration (Fig. 1b). Whereas pure CdSe NCs show no sub-bandgap luminescence, addition of $\mathrm{Cu}^{+}$ induces a broad defect luminescence in the sub-bandgap 1.4$1.8 \mathrm{eV}$ region. With increasing $\mathrm{Cu}^{+}$feed concentration, the defect luminescence increased in intensity, as would be consistent with an increase in copper impurities and associated defects prior to the onset of cation exchange. The defect luminescence intensity reached a maximum around the critical $\mathrm{Cu}^{+}$concentration, beyond which it started decreasing. Such a decrease is expected from the sharp onset of cation exchange, because the transition to $\mathrm{Cu}_{2} \mathrm{Se}$ involves mutual annihilation of $\mathrm{Cu}^{+}$impurities with $\mathrm{Cd}^{2}+$ vacancies, expressed in Kroger-Vink notation as:

$$
2 \cdot \mathrm{Cu}_{\mathrm{i}}^{\cdot}+\mathrm{V}_{\mathrm{Cd}^{\mathrm{x}}}+\mathrm{Se}^{2-} \rightleftharpoons \mathrm{Cu}_{2} \mathrm{Se} .
$$

Thus, doping of a CdSe NC with $\mathrm{Cu}^{+}$appears to be a critical precursor to the onset of cation exchange (Fig. $4 \mathrm{a}$ ). The strongest 

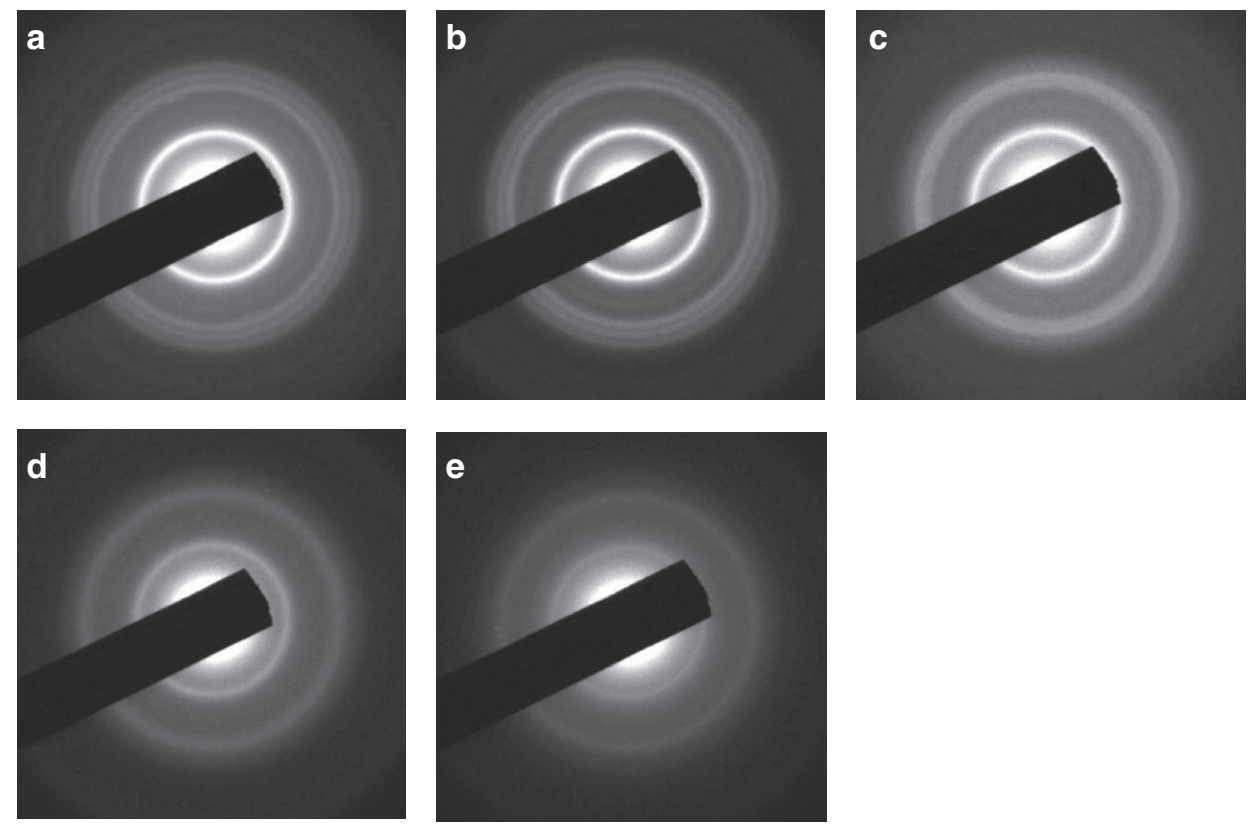

Figure 3 | Electron diffraction reveals the sharp onset of cation exchange. For NCs subject to $0 \mathrm{Cu}^{+}$ions per $\mathrm{NC}$ and $384 \mathrm{Cu}^{+}$ions per $\mathrm{NC}$ respectively $(\mathbf{a}, \mathbf{b})$, selected area electron diffraction patterns are indicative of the CdSe wurtzite (cadmoselite) structure. As shown in Table 1, the innermost ring at $3.55 \AA$ is a combination of the (100), (002) and (101) planes, which are Debye-Scherrer broadened into one ring. The three outer rings, measured at 2.15, 2.00 and $1.88 \AA$, match those for the (100), (103) and (200) planes of cadmosellite. In addition, there is a ring corresponding to the (102) plane, at a lattice spacing of $2.55 \AA$. These features are seen for samples (a-c). At 1,317 Cu${ }^{+}$ions per NC (in slight excess of the stoichiometric amount needed for full conversion), the tetragonal $\mathrm{Cu}_{2} \mathrm{Se}$ phase (bellidoite) is seen (e). The highest intensity reflections include ones at 3.46 $\AA$ for the (113), (311) and (222) planes, $2.04 \AA$ for the (404) plane, and $1.76 \AA$ for the (226) and (533) plane. The diffraction features of $\mathrm{Cu}_{2} \mathrm{Se}$ seem to first appear at $549 \mathrm{Cu}^{+}$ions per $\mathrm{NC}$ (c), overlapped with CdSe diffraction features. As exchange progresses, CdSe diffraction features wane, until a pure bellidoite diffraction pattern is obtained for the fully converted sample. All d-spacings are determined relative to the (111) reflection of an Au standard taken under the same conditions. Samples were prepared by a slow titration of CdSe NCs in toluene with increasing amounts of a methanolic solution of $\mathrm{Cu}^{+}$, similar to the titration experiments described in Methods. At various points in the titration, a small aliquot was drop-casted onto an ultrathin carbon transmission electron microscope grid and washed with methanol several times. The total amount of $\mathrm{Cu}^{+}$added is indicated in Table 1 in the form of number of ions per $\mathrm{NC}$, estimated as described in Methods.

\section{Table 1 | d-spacings obtained from electron diffraction of NCs at various stages of $\mathrm{Cu}^{+}$exchange.}

\begin{tabular}{|c|c|c|c|c|}
\hline $\begin{array}{l}\text { Sample A } \\
0 \mathrm{Cu}^{+} \text {per } \\
\mathrm{NC}(\mathrm{A})\end{array}$ & $\begin{array}{c}\text { Sample B } \\
384 \mathrm{Cu}^{+} \\
\text {per NC (A) }\end{array}$ & $\begin{array}{c}\text { Sample C } \\
549 \mathrm{Cu}^{+} \\
\text {per NC (A) }\end{array}$ & $\begin{array}{c}\text { Sample D } \\
659 \mathrm{Cu}^{+} \\
\text {per NC (A) }\end{array}$ & $\begin{array}{c}\text { Sample E } \\
1,317 \mathrm{Cu}^{+} \\
\text {per NC (A) }\end{array}$ \\
\hline 3.57 & 3.57 & 3.54 & 3.47 & 3.46 \\
\hline 2.55 & 2.55 & 2.57 & 2.04 & 2.04 \\
\hline 2.17 & 2.17 & 2.17 & 1.77 & 1.76 \\
\hline 2.02 & 2.01 & 2.05 & & \\
\hline 1.88 & 1.89 & 1.79 & & \\
\hline
\end{tabular}

affirmation comes from two observations in Fig. 2a: first, the point in the titration where the defect luminescence intensity reaches a maximum is coincident with the point of onset of cation exchange. Second, the plot of defect luminescence intensity as a function of feed $\mathrm{Cu}^{+}$concentration resembles the derivative of the sigmoidal titration curve.

\section{Discussion}

The extended latent region below the critical concentration suggests that the initial incorporation of $\mathrm{Cu}^{+}$into the lattice is a relatively low-probability process. However, initial doping of a NC can occur stochastically, possibly at surface or defect sites of a NC. These initial doping events activate the NC for further doping. Each doping event enhances the affinity of the NC for a subsequent doping event and so on, until the $\mathrm{NC}$ reaches a critical state where it is likely to take up every available $\mathrm{Cu}^{+}$ion, resulting in a cascade of $\mathrm{Cu}^{+}$incorporation and an abrupt phase transition from a low-doped CdSe $\mathrm{NC}$ to $\mathrm{Cu}_{2} \mathrm{Se}$. In other words, a $\mathrm{NC}$ already doped with $\mathrm{Cu}^{+}$is much more likely to take up additional $\mathrm{Cu}^{+}$and make a sharp transition to $\mathrm{Cu}_{2} \mathrm{Se}$, albeit at the expense of another $\mathrm{NC}$ which has acquired none or relatively few dopants. This bias represents positive co-operativity between 'sites' on the same NC.

When energetic aspects of such positive co-operative behaviour were included in a lattice site model (Methods section $)^{25}$, we were able to simulate all our experimental observations (Fig. 2b). We started with an ensemble of CdSe NCs, each with 20 bivalent sites for $\mathrm{Cu}^{+}$. For the first doping event within any NC, the magnitude of the free energy change $\Delta G$ was assumed to be small, making it a low probability event. However, each doping event increased, by an amount $\Delta g$, the free energy change resulting from subsequent doping on the same NC. Thus, it is energetically more and more favourable for a NC to incorporate $\mathrm{Cu}^{+}$ions. This simple model reproduced the strongly sigmoidal response of the $\mathrm{NC}$ ensemble to the added $\mathrm{Cu}^{+}$. There was an extended latent region during which only a tiny fraction of NCs made a transition to $\mathrm{Cu}_{2} \mathrm{Se}$. Most of the NCs made a transition over a narrow window around a critical $\mathrm{Cu}^{+}$feed concentration. The simulations also reproduced the rise in the number of defects in the ensemble with increasing $\mathrm{Cu}^{+}$feed concentration. The number of defects reached a maximum around the critical concentration, following which it gradually decreased to zero. If there were no such positive co-operative bias (that is, if every site 

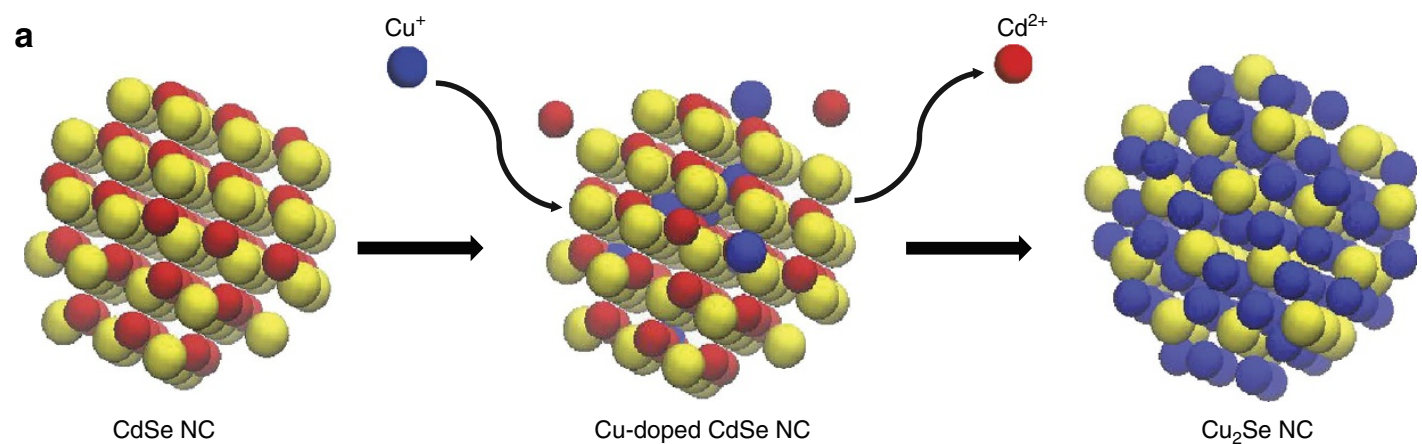

b

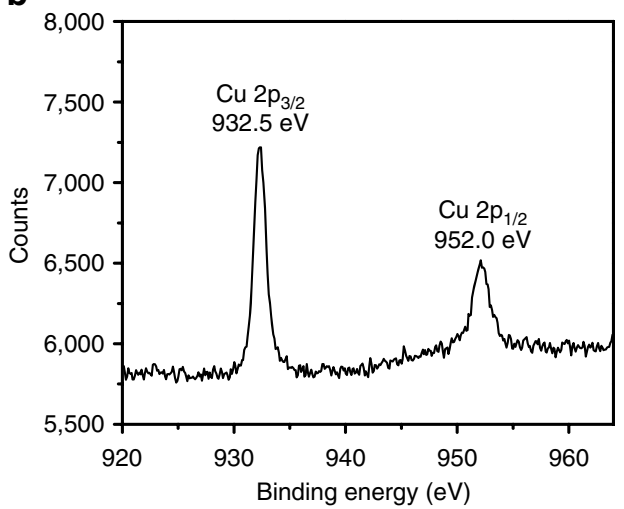

c

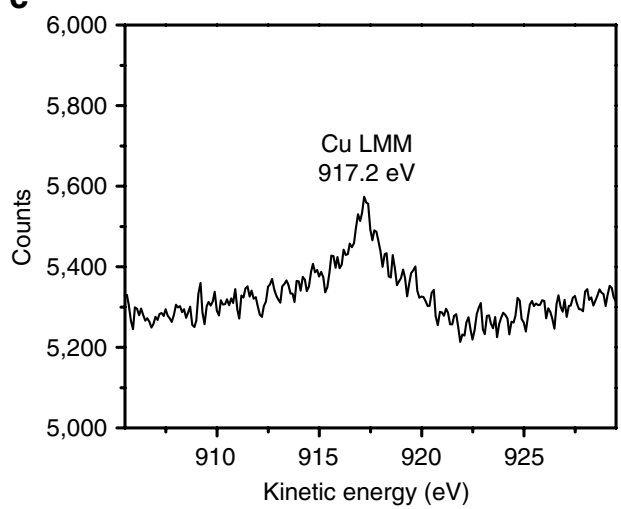

Figure 4 | Cation exchange is preceded by doping with $\mathbf{C u}^{+}$. A model of a CdSe wurtzite crystal (a) with ordered cadmium (red) and selenium (yellow) atoms. Below the critical concentration, the CdSe NC uptakes copper ions (blue) in the form of interstitial dopants, in turn creating C $\mathrm{d}^{2+}$ vacancies within the NC for charge compensation. These impurities and vacancies are manifested in the emergence of defect luminescence in Fig. $1 \mathrm{~b}$. Initial doping events can greatly enhance the affinity of the $\mathrm{NC}$ for additional $\mathrm{Cu}^{+}$, eventually triggering a sharp transition to the $\mathrm{Cu}_{2} \mathrm{Se}$ state. The formation of a $\mathrm{Cu}_{2} \mathrm{Se} \mathrm{NC}$ involves the mutual annihilation of $\mathrm{Cu}^{+}$dopants and $\mathrm{Cd}^{2}+$ vacancies, resulting in the decrease in defect luminescence seen above the critical concentration. High-resolution XPS of NCs treated with a copper concentration of $325 \mathrm{Cu}^{+}$ions per NC are shown (b). NCs were thoroughly washed with methanol to remove excess $\mathrm{Cu}^{+}$before measurements. The fitted peak position of the $\mathrm{Cu} 2 \mathrm{p}_{3 / 2}$ band ( 932.5 eV) is indicative of copper

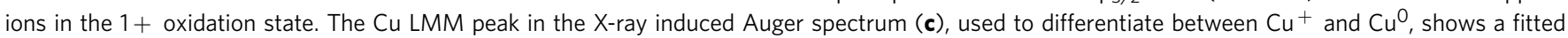
peak position of $917.2 \mathrm{eV}$, characteristic of $\mathrm{Cu}^{(1+)}$. Additional XPS data are shown in the Supplementary Information.

in the CdSe ensemble had a similar affinity for $\mathrm{Cu}^{+}$regardless of whether the site is on an already doped $\mathrm{NC}$ or an undoped one), the exchange process would be a diffusion-limited one. In such a case, each NC would exchange gradually via a continuum of intermediary $\mathrm{CdSe} / \mathrm{Cu}_{2} \mathrm{Se}$ heterostructure states. The titration curve would be Langmuirian $\left(n_{\mathrm{H}}=1\right)$ rather than sigmoidal $\left(n_{\mathrm{H}}>1\right)$.

Thus, a positive co-operative model, similar to one employed in biomacromolecular systems ${ }^{26}$, captures the observed behaviour of the cation exchange process. Nevertheless it still leaves open the question of the atomistic mechanism for co-operativity. In haemoglobin, oxygen binding at one of the heme subunits leads to a conformational change in the neighbouring subunits, which increases the affinity of the neighbour site for oxygen binding, a phenomenon known as allostery ${ }^{22}$. Allosteric coupling between lattice sites of a NC can originate from a few different structural factors. As described earlier, owing to charge compensation, $\mathrm{Cu}^{+}$ doping results in the creation of $\mathrm{Cd}^{2}+$ vacancies, one vacancy for every two interstitial $\mathrm{Cu}^{+}$impurities. These lattice vacancies provide hopping sites for enhanced in-diffusion of additional $\mathrm{Cu}^{+}$ions into the NC and out-diffusion of $\mathrm{Cd}^{2+}$ ions from the NC. Second, the local negative polarization at a $\mathrm{Cd}^{2+}$ vacancy site could electrostatically attract other positively charged $\mathrm{Cu}^{+}$ ions. Third, inclusion of interstitial impurities and formation of vacancies would strain the NC lattice greatly enhancing the thermodynamic driving force for $\mathrm{Cu}^{+}$substitutions and structural transition to $\mathrm{Cu}_{2} \mathrm{Se}$. Such mechanisms have been found to be operative in super-ionic solids ${ }^{27}$.

Cation exchange with another common monovalent cation $\mathrm{Ag}^{+}$showed similar results: a sigmoidal titration curve and a maximum in the defect luminescence intensity around the critical concentration (Fig. 5), indicating that the co-operative mechanism of cation exchange is not specific just to $\mathrm{Cu}^{+}$. The $\mathrm{Ag}$ system appears to be less co-operative than the $\mathrm{Cu}$ system: the titration curve is not as steep. The Hill fit shows a value of $n_{\mathrm{H}}=3.2$ smaller than for $\mathrm{Cu}^{+}$. The plot of the defect luminescence intensity as a function of feed concentration does not show a sharp peak, rather a plateau. Although the physical reason for this difference is not understood, it could be due to the significantly larger size of $\mathrm{Ag}^{+}$ than $\mathrm{Cu}^{+}$. The larger $\mathrm{Ag}^{+}$ions may have a lower propensity to occupy interstitial sites and substitutional doping may be more prevalent than in the $\mathrm{Cu}^{+}$case.

Although positive co-operativity between sites of individual NCs explains all our observed results, a related mechanism is worth considering. It is possible for initial $\mathrm{Cu}$ (or Ag) impurities formed within a NC to cluster together into a critical-size nucleus, initiating spontaneous growth of the $\mathrm{Cu}_{2} \mathrm{Se}$ (or $\mathrm{Ag}_{2} \mathrm{Se}$ ) phase within the NC. This is reminiscent of the photographic development of silver iodide, where a latent four-atom nucleation cluster triggers rapid spontaneous growth of $\mathrm{Ag}^{28-30}$. Such a nucleation-growth mechanism could give rise to the observed two-state behaviour and the sharp sigmoidal transition. However, 


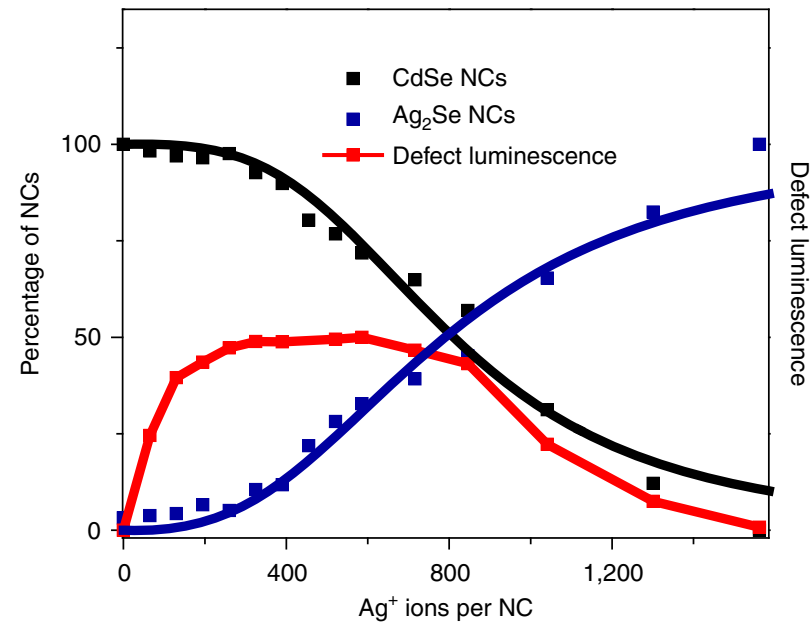

Figure 5 | Co-operativity in the cation exchange of CdSe NCs with $\mathbf{A g}^{+}$. A plot of the fraction of NCs in the CdSe state (black dots) and those in the $\mathrm{Ag}_{2} \mathrm{Se}$ state (blue) as a function of added $\mathrm{Ag}^{+}$is sigmoidal, indicative of co-operative behaviour. Below a critical concentration of $\sim 500 \mathrm{Ag}^{+} / \mathrm{NC}$, very few NCs undergo a transition to the $\mathrm{Ag}_{2}$ Se state. $\mathrm{A}$ fit to the Hill plot (solid black line, $R=0.973$ ) yields a co-operativity factor of $n_{H}=3.2$. The fraction of NCs in the CdSe state was determined from the height of the $\mathrm{CdSe}$ excitonic peak and the fraction in the $\mathrm{Ag}_{2} \mathrm{Se}$ state was determined from the $\mathrm{Ag}_{2} \mathrm{Se}$ band-to-band absorption at $1.82 \mathrm{eV}$. The absorption and PL data is shown in Supplementary Fig. S5, and the procedure for analysis is described in Methods. The rise in the $\mathrm{Ag}_{2} \mathrm{Se}$ fraction tracks well with the drop in the CdSe fraction. The defect luminescence intensity (red), integrated across its broad band, increases as $\mathrm{Ag}^{+}$is added, until it reaches a maximum around the critical concentration, beyond which it decreases, until it reaches zero.

the topotactic nature of cation exchange $e^{31}$ is not compatible with a nucleation-driven mechanism. In the latter mechanism, the final nanostructure morphology would be dictated by the growing nucleus, rather than by the starting ionic template.

A common feature across many co-operative systems is that each binding event pre-organizes other sites for binding, thereby reducing the number of non-productive configurations and resulting in greatly enhanced reaction rates. For instance, cooperativity allows a protein to fold rapidly within milliseconds, rather than requiring the protein to sample all possible configurations. The latter would need a time longer than the age of the universe. It is possible that cation exchange reactions in NCs are driven to completion due to the strongly co-operative nature of the transition.

Akin to co-operativity between molecular units in the biological world, co-operativity between atoms may be ubiquitous in the solid state, where there is no dearth of multi-site systems. Formation of defects, interfaces or strain may provide the required inter-site interactions. Co-operative behaviour in solidstate systems needs closer attention, especially in relation with closely linked processes such as phase transitions and nucleation $^{32,33}$ and due to the utility of co-operative phenomena in predicting and controlling materials' transformation outcomes. Nonlinear behaviour induced by co-operativity may allow the design of sensors with tight regulation.

\section{Methods}

Synthesis of CdSe NCs. The procedure for synthesis of CdSe NCs was adapted from Manna and co-workers ${ }^{34}$. Three grams of trioctylphosphine oxide, $0.280 \mathrm{~g}$ of octadecylphosphonic acid (ODPA) and $0.060 \mathrm{~g}$ of cadmium oxide were mixed in a
$25 \mathrm{ml}$ three neck flask, heated to $150^{\circ} \mathrm{C}$ and exposed to vacuum for $1 \mathrm{~h}$. Under argon, the solution was heated to $320^{\circ} \mathrm{C}$ and held at that temperature until the solution was optically clear, indicating complete dissolution of cadmium oxide. At this point, $1.5 \mathrm{~g}$ of tri- $n$-octylphosphine (TOP) was injected into the flask. The flask was heated to $370{ }^{\circ} \mathrm{C}$ and a TOP-Se solution $(0.058 \mathrm{~g}$ of selenium powder and $0.360 \mathrm{~g}$ of TOP) was injected into the flask. The reaction time was $1 \mathrm{~min}$ for obtaining CdSe NCs of $4 \mathrm{~nm}$ diameter. After the synthesis, the NCs were repeatedly precipitated with methanol followed by re-dissolution in toluene.

Stoichiometry calculations for cation exchange titrations. For titrations, the initial concentration of $\mathrm{Cd}^{2+}$ ions from the CdSe NCs in each reaction cuvette was determined. From the position of the first exciton, $\lambda$, the diameter, $D$, of the NC and the extinction coefficient were calculated using the equations ${ }^{35}$ :

$$
\begin{gathered}
D=\left(1.6122 \times 10^{-9}\right) \lambda^{4}-\left(2.6575 \times 10^{-6}\right) \lambda^{3}+\left(1.6242 \times 10^{-3}\right) \lambda^{2} \\
-(0.4277) \lambda+(41.57) \\
\epsilon=5857(D)^{2.65} .
\end{gathered}
$$

The NCs used in all the titrations had a diameter of $\sim 4 \mathrm{~nm}$. The extinction coefficient $\epsilon$ and the absorbance at the first exciton were used to determine the concentration of NCs. From the diameter, the volume and mass of NCs was calculated, from which the total mass and molar amount of $\mathrm{Cd}^{2}+$ ions in each CdSe NC solution was determined. All titrations were done with approximately equal concentrations of CdSe NCs. Each $\mathrm{Cu}^{+}$titration involved $4.8 \times 10^{-6} \mathrm{~mol}$ of $\mathrm{Cd}^{2+}$ ions being exchanged with one stoichiometric equivalent of $\mathrm{Cu}^{+}$ions $\left(9.6 \times 10^{-6} \mathrm{~mol}\right.$ of $\left.\mathrm{Cu}(\mathrm{I}) \mathrm{PF}_{6}\right)$ dissolved in $200 \mu \mathrm{l}$ of methanol. The details for each incremental addition in the titration is given in Supplementary Table S2. The rightmost column of this table was used as the $x$ axis value for the titration plots shown in Fig. 2.

Cation exchange procedure. All exchange reactions were carried out in septacapped $3.5 \mathrm{ml}$ quartz cuvettes. In an oxygen and moisture-free glove box, CdSe NCs were dispersed in $3.0 \mathrm{ml}$ of toluene. Initial $\mathrm{Cd}^{2}+$ molar amounts were calculated as described above to be $4.8 \times 10^{-6} \mathrm{~mol} \mathrm{Cd}^{2+}$. In total, $9.6 \times 10^{-6} \mathrm{~mol}$ of tetrakis(acetonitrile)copper(I) hexafluorophosphate $\left(\mathrm{Cu}(\mathrm{I}) \mathrm{PF}_{6}\right)$ in $200 \mu \mathrm{l}$ of methanol was added during each titration. NC samples were prepared in the glove box in air-free conditions and kept in air-free cuvettes during the exchange reactions. Additions took place outside of the glove box. A solution of $\mathrm{Cu}(\mathrm{I}) \mathrm{PF}_{6}$ in methanol, prepared in the glove box and kept sealed during the experiment, was sequentially added to the reaction cuvette with a microliter syringe, taking care that the solution or sample were not exposed to air. Samples were stirred vigorously after each addition and the solution was allowed to equilibrate while stirring for $\sim 1 \mathrm{~h}$, which is a time much in excess of the typical reaction time for complete cation exchange $(<1 \mathrm{~s})$. Absorption and photoluminescence $(\mathrm{PL})$ spectra were then taken.

Optical spectroscopy measurements. Absorption spectra of exchanged CdSe NCs were collected on a Shimadzu UV-3600 scanning spectrophotometer with a $1 \mathrm{~nm}$ step size with toluene as a reference solvent. PL spectra of NCs were taken in toluene on a Cary Eclipse fluorimeter. Spectra were taken in the $650-900 \mathrm{~nm}$ wavelength range, where defect luminescence appears. All PL measurements were performed using the same slit width $(5 \mathrm{~nm})$, scan speed $(120 \mathrm{~nm} / \mathrm{min})$, excitation wavelength $(450 \mathrm{~nm})$ and photomultiplier tube voltage.

Analysis of absorption and PL spectra. The total volume change over the entire titration was $<10 \%$, therefore correction of spectra for volumetric dilution was not necessary. The acquired absorbance spectra contain absorption from both unexchanged CdSe NCs and formed $\mathrm{Cu}_{2} \mathrm{Se} \mathrm{NCs}$. The absorption contribution of the formed $\mathrm{Cu}_{2} \mathrm{Se}$ NCs was subtracted from the acquired spectra to isolate the absorption of unexchanged CdSe NCs as follows:

$$
\begin{aligned}
& \text { Corrected spectrum } \\
& =\text { Uncorrected spectrum } \\
& \quad-\left(\mathrm{Cu}_{2} \mathrm{Se} \text { spectrum } \times \frac{\text { Absorbance of uncorrected spectrum at } 1.82 \mathrm{eV}}{\text { Absorbance of } \mathrm{Cu}_{2} \mathrm{Se} \text { spectrum at } 1.82 \mathrm{eV}}\right)
\end{aligned}
$$

Here, we use the fact that at $1.82 \mathrm{eV}$, the absorption contribution from CdSe is negligible. The absorbance $A$ at the first exciton peak of the corrected spectrum is proportional to the concentration of unexchanged CdSe NCs in solution as per Beer-Lambert's law. This absorbance $A$ normalized to a value of $100 \%$ at $0 \%$ exchange is equal to the percentage of CdSe NCs that is still unexchanged. This percentage was plotted as a function of $\mathrm{Cu}^{+}$added in Fig. 2a. The absorbance of $\mathrm{Cu}_{2} \mathrm{Se}$ at $1.82 \mathrm{eV}$ (that is, above its bandgap absorption onset) can be used to estimate the relative concentration of $\mathrm{Cu}_{2} \mathrm{Se}$ NCs produced over the course of the titration. The absorbance at $1.82 \mathrm{eV}$ normalized to a value of $100 \%$ at full exchange is equal to the percentage of $\mathrm{Cu}_{2} \mathrm{Se} \mathrm{NCs}$ formed. This percentage was also plotted in Fig. $2 \mathrm{a}$ as a function of $\mathrm{Cu}^{+}$feed concentration. 
PL spectra were corrected by removing the contribution from the excitonic PL of CdSe NCs as follows:

$$
\begin{aligned}
& \text { Corrected spectrum } \\
& =\text { Uncorrected spectrum }-(\text { unexchanged CdSe spectrum } \\
& \left.\quad \times \frac{\text { PL intensity of uncorrected spectrum at } 650 \mathrm{~nm}}{\text { PL intensity of unexchanged CdSe at } 650 \mathrm{~nm}}\right) .
\end{aligned}
$$

After this correction, the defect luminescence bands were integrated between 650 and $900 \mathrm{~nm}$. The integrated defect PL signal was plotted in Fig. 2a as a function of $\mathrm{Cu}^{+}$feed concentration. We also performed a control experiment to confirm that methanol exposure alone does not give rise to defect PL in the NCs (Supplementary Fig. S3).

Electron microscopy. High-resolution transmission electron microscopy (HRTEM) images were taken using a JEOL $2010 \mathrm{LaB}_{6}$ operating at $200 \mathrm{kV}$. Highangle annular dark-field scanning electron transmission microscopy (HAADFSTEM) images were acquired on a JEOL $2010 \mathrm{~F}$ at $200 \mathrm{kV}$ with a $0.5 \mathrm{~nm}$ size beam. Samples of NCs were prepared by drop-casting NCs in toluene on an ultrathin carbon grid followed by repeated washing with methanol.

NC morphology. HRTEM and HAADF-STEM images (Supplementary Figs S4 and S5) show the morphology of NCs as a function of the feed $\mathrm{Cu}^{+}$concentration in $\mathrm{Cu}^{+}$ions per NC. Samples were made from adding $\mathrm{Cu}(\mathrm{I}) \mathrm{PF}_{6}$ in $200 \mu$ l methanol to CdSe NCs in toluene. The NCs were drop-casted from this toluene:methanol mixture onto a $\mathrm{Cu}$ grid, followed by washing with methanol to remove excess $\mathrm{Cu}$ and $\mathrm{Cd}^{2+}$ resulting from exchange. Images show preservation of size and morphology throughout the exchange process. NC sizing analysis was performed on HAADF-STEM images. Lattice fringes in HRTEM show that NCs maintain crystallinity throughout the exchange process.

Elemental analysis. ICP-OES (inductively coupled plasma optical emission spectrometry) was performed on a Perkin-Elmer Optima 2000 DV instrument. To prepare samples, NCs were repeatedly washed and precipitated with methanol to remove excess ions in solution. Approximately $1 \mathrm{mg}$ of each sample was digested with $16 \mathrm{M} \mathrm{HNO}_{3}$ and subject to ICP-OES analysis for determination of the molar ratios of $\mathrm{Cd}, \mathrm{Cu}$ and $\mathrm{Se}$ (Supplementary Table $\mathrm{S} 1$ ).

Exchange with $\mathbf{A g}^{+}$. Titration experiments with $\mathrm{Ag}^{+}$were performed in an identical manner as those with $\mathrm{Cu}^{+}$. Initial $\mathrm{Cd}^{2+}$ molar amounts were calculated as described above to be $4.4 \times 10^{-6} \mathrm{~mol}$ of $\mathrm{Cd}^{2+}$ in each cuvette. In total, $8.9 \times 10^{-6} \mathrm{~mol}$ of silver nitrate $\left(\mathrm{AgNO}_{3}\right)$, corresponding to one stoichiometric equivalent, was the calculated amount to be added over the entire titration Slightly more was actually found to be needed $\left(1.1 \times 10^{-5} \mathrm{~mol}\right.$ of $\mathrm{AgNO}_{3}$ in $240 \mu \mathrm{l}$ of methanol) to ensure complete disappearance of the first exciton peak of CdSe

Absorption and PL spectra were corrected and processed using the same methods described earlier. Uncorrected absorption spectra (Supplementary Fig. S6a) were baseline corrected (Supplementary Fig. S6c) to account for absorption of $\mathrm{Ag}_{2} \mathrm{Se}$ NCs. The CdSe exciton peak heights were then analysed to estimate the percentage of NCs exchanged as a function of $\mathrm{Ag}^{+}$added (Fig. 5). The PL spectra (Supplementary Fig. S6b) were corrected (Supplementary Fig. S6d) to remove the band-edge PL. Following correction, the defect luminescence band was integrated to obtain the trend in defect luminescence intensity as a function of $\mathrm{Ag}^{+}$added (Fig. 5).

X-ray photoelectron spectroscopy. XPS analysis was performed on NCs exchanged with 0,325 and $651 \mathrm{Cu}^{+}$ions per NC. A Kratos Axis ULTRA instrument was used, which uses a monochromatized Al $K_{\alpha} \mathrm{X}$-ray source of $15 \mathrm{kV}$ $(\sim 10 \mathrm{~mA})$. The instrument consisted of separate specimen transfer and analysis chambers, typically operated at $5 \mathrm{E}-07$ and $1 \mathrm{E}-09$ Torr, respectively. The $\mathrm{Cu} 2 \mathrm{p}_{3 / 2}$ spectrum was fitted with $30 \%$ Gaussian/70\% Lorentzian peak shapes. C $1 \mathrm{~s}$ peak was used as a reference. The samples subject to 325 and $651 \mathrm{Cu}^{+}$ions per NC (Fig. $4 \mathrm{~b}$ and Supplementary Fig. S1c respectively) show a $\mathrm{Cu} 2 \mathrm{p}_{3 / 2}$ with a fitted peak position of $932.5 \mathrm{eV}$, which suggests a $1+$ oxidation state. To distinguish between $\mathrm{Cu}^{0}$ and $\mathrm{Cu}^{+}$, high-resolution spectra were collected around the X-rayinduced $\mathrm{Cu}$ LMM Auger peak (shown in Fig. $4 \mathrm{c}$ and Supplementary Fig. S1d). The peak position of $917.2 \mathrm{eV}$ (K.E.) corresponds to $\mathrm{Cu}^{(1+)}$. The absence of the $\mathrm{Cu}^{(1+)}$ peak in the $\mathrm{Cu} 2 \mathrm{p}_{3 / 2}$ (Supplementary Fig. S1a) and Cu LMM (Supplementary Fig. $\mathrm{S} 1 \mathrm{~b})$ spectra for the unexchanged sample confirms that there is no $\mathrm{Cu}$ in the original CdSe NCs.

Theoretical model. We simulated the response of NCs using a lattice-site model. Each NC was assumed to have a size of $n=20$ sites, each of which can incorporate two $\mathrm{Cu}^{+}$ions. Co-operativity was introduced in this model by requiring that doping takes place sequentially: only when a doping event has taken place at the first site in the NC does a second site become available in that NC, and so on.

$$
\begin{array}{cccc}
(\mathrm{CdSe})_{n}+2 \mathrm{Cu}^{+} & \rightleftharpoons & (\mathrm{CdSe})_{n-1}\left(\mathrm{Cu}_{2} \mathrm{Se}\right)+\mathrm{Cd}^{2+} & \\
M & M L & k_{1} \\
(\mathrm{CdSe})_{n-1}\left(\mathrm{Cu}_{2} \mathrm{Se}\right)+2 \mathrm{Cu}^{+} & \rightleftharpoons & (\mathrm{CdSe})_{n-2}\left(\mathrm{Cu}_{2} \mathrm{Se}\right)_{2}+\mathrm{Cd}^{2+} & \\
M L & & M L_{2} & k_{2} \\
(\mathrm{CdSe})_{n-2}\left(\mathrm{Cu}_{2} \mathrm{Se}\right)_{2}+2 \mathrm{Cu}^{+} & \rightleftharpoons & (\mathrm{CdSe})_{n-3}\left(\mathrm{Cu}_{2} \mathrm{Se}\right)_{3}+\mathrm{Cd}^{2+} & \\
M L_{2} & & M L_{3} & k_{3} \\
\vdots & \vdots & \vdots \\
(\mathrm{CdSe})\left(\mathrm{Cu}_{2} \mathrm{Se}\right)_{n-1}+2 \mathrm{Cu}^{+} & \rightleftharpoons & \left(\mathrm{Cu}_{2} \mathrm{Se}\right)_{n}+\mathrm{Cd}^{2+} & \\
M L_{n-1} & & M L_{n} & k_{n}
\end{array}
$$

$$
\text { . }
$$

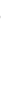

here $M, M L, M L_{2}$ and so on are relative concentrations of $\mathrm{NC}$ complexes from CdSe through to $\mathrm{Cu}_{2} \mathrm{Se}$ and $k_{1}, k_{2}, k_{3}$ and so on are association constants for each sequential doping step.

$$
k_{1}=\exp ^{-\Delta G / k T} .
$$

We assumed that the association constant of the first doping event $k_{1}$ has a relatively small magnitude $\left(10^{-2} \mathrm{M}^{-1}\right)$. However, the first step enhances the free energy for the second step by an amount $\Delta g$, and the second step enhances that of the third one and so on. Therefore,

$$
\begin{gathered}
k_{2}=\exp ^{-(\Delta G+\Delta g) / k T} \\
k_{3}=\exp ^{-(\Delta G+2 \Delta g) / k T} \\
\vdots \\
k_{n}=\exp ^{-(\Delta G+(n-1) \Delta g) / k T}
\end{gathered}
$$

or,

$$
k_{2} / k_{1}=k_{3} / k_{2}=k_{4} / k_{3}=\cdots=s k_{n} / k_{n-1}=a
$$

where $a=\exp ^{-\Delta g / k T}$. As $\Delta g$ is negative, $a$ is a positive integer, which we term as the interaction parameter. The magnitude of $a$ depends directly on the magnitude of free energy stabilization $(\Delta g)$ relative to thermal energy $(k T)$. For instance, a 0.1 $\mathrm{eV}$ stabilization can result in $a \sim 55$, which was a typical value used in the simulations. A higher value of $a$ would allow the transition to be achieved over a narrower concentration window for the same value of $k_{1}$.

For a free $\mathrm{Cu}^{+}$concentration $c$ and $\mathrm{Cd}^{2+}$ concentration $d$, concentrations of the different $\mathrm{NC}$ complexes can be given in terms of the concentration of unexchanged CdSe NCs $M$ as:

$$
\begin{array}{cc}
M L & =k_{1} \cdot M \cdot c^{2} / d \\
M L_{2} & =k_{2} \cdot M L \cdot c^{2} / d \\
M L_{3} & =k_{3} \cdot M L_{2} \cdot c^{2} / d \\
\vdots & \vdots \\
M L_{n} & =k_{n} \cdot M L_{n-1} \cdot c^{2} / d .
\end{array}
$$

In general,

$$
M L_{i}=M / a(k \cdot a)^{i}
$$

where we define a dimensionless $k$ :

$$
k=k_{1} \cdot c^{2} / d .
$$

The fraction of NCs in the pure CdSe state is given by:

$$
M / M_{T}=a /\left\{a+\sum_{i=1}^{n}(k \cdot a)^{i}\right\}
$$

where

$$
M_{T}=M+M L+M L_{2}+\cdots+M L_{n}=M\left\{1+1 / a \sum_{i=1}^{n}(k \cdot a)^{i}\right\} .
$$

The fraction of NCs in the $\mathrm{Cu}_{2} \mathrm{Se}$ state is given by:

$$
M L_{n} / M_{T}=(k \cdot a)^{n} /\left\{a+\sum_{i=1}^{n}(k \cdot a)^{i}\right\}
$$

$1-M L_{n} / M_{T}$ gives the fraction of NCs that are either purely CdSe or Cu-doped CdSe. The total concentration of $\mathrm{Cu}^{+}$dopants is given by:

$$
\begin{aligned}
c_{d} & =2\left\{M L+2 \cdot M L_{2}+\cdots+(n-1) \cdot M L_{n}\right\} / M_{T} \\
& =2\left\{\sum_{i=1}^{n-1} i(k \cdot \mathrm{a})^{i}\right\} /\left\{a+\sum_{i=1}^{n}(k \cdot \mathrm{a})^{i}\right\} .
\end{aligned}
$$

Note in the above expression $\mathrm{Cu}^{+}$ions in $\mathrm{Cu}_{2} \mathrm{Se}$ state are not included as they are no longer in the form of dopants and do not contribute to defect luminescence. Free $\mathrm{Cu}^{+}$ions in solution are not included either. $c_{d}$ is also equal to two times the concentration of $\mathrm{Cd}^{2+}$ vacancies. The concentration of $\mathrm{Cu}^{+}$incorporated in the $\mathrm{NCs}$, including in the form of $\mathrm{Cu}_{2} \mathrm{Se}$, is given by:

$$
c_{b}=2\left\{\sum_{i=1}^{n} i(k \cdot \mathrm{a})^{i}\right\} /\left\{a+\sum_{i=1}^{\mathrm{n}}(k \cdot \mathrm{a})^{i}\right\} .
$$


The free $\mathrm{Cd}^{2}+$ concentration is given by:

$$
d=c_{b} / 2 \text {. }
$$

The free $\mathrm{Cu}^{+}$concentration is given by:

and the total $\mathrm{Cu}^{+}$concentration as

$$
c_{f}=\sqrt{c_{b} \cdot k / 2 k_{1}}
$$

$$
c_{t}=c_{b}+c_{f}
$$

\section{References}

1. Hill, A. V. The possible effects of the aggregation of the molecule of hemoglobin on its dissociation curves. J. Physiol. 40, iv-vii (1910).

2. Akers, G. K., Doyle, M. L., Myers, D. \& Daugherty, M. A. Molecular code for cooperativity in hemoglobin. Science 255, 54-63 (1992).

3. Whitty, A. Cooperativity and biological complexity. Nat. Chem. Bio. 4, 435-439 (2008).

4. Shank, E. A., Cecconi, C., Dill, J. W., Marqusee, S. \& Bustamante, C. The folding cooperativity of a protein is controlled by its chain topology. Nature 465, 637-640 (2010).

5. Prince, R. B., Saven, J. G., Wolynes, P. G. \& Moore, J. S. Cooperative conformational transitions in phenyl ethylene oligomers: chain-length dependence. J. Am. Chem. Soc. 121, 3114-3121 (1999).

6. Hunter, C. A. \& Anderson, H. L. What is Cooperativity? Angew. Chem. Int. Ed. 48, 7488-7499 (2009)

7. Dukes, A. D., McBride, J. R. \& Rosenthal, S. J. Synthesis of the magic-sized $\mathrm{CdSe}$ and CdTe nanocrystals with diisooctylphosphinic acid. Chem. Mater. 22, 6402-6408 (2010).

8. Don, H. S., Hughes, S. M., Yin, Y. \& Alivisatos, A. P. Cation exchange reactions in Ionic Nanocrystals. Science 306, 1009-1012 (2004).

9. Robinson, R. D. et al. Spontaneous superlattice formation in nanorods through partial cation exchange. Science 317, 355-358 (2007).

10. Pollok, K., Putnis, C. V. \& Putnis, A. Mineral replacement reactions in solid solution-aqueous solution systems: Volume changes, reactions paths and endpoints using the example of model salt systems. Am. J. Sci. 311, 211-236 (2011).

11. Chan, E. M. et al. Millisecond kinetics of nanocrystal cation exchange using microfluidic x-ray absorption spectroscopy. J. Phys. Chem. A. 111, 12210-12215 (2007).

12. Miszta, K. et al. Hierarchical self-assembly of suspended branched colloidal nanocrystals into superlattice structures. Nat. Mater. 10, 872-876 (2011).

13. Kovalenko, M. V. Quasi-seeded growth of ligand-tailored PbSe nanocrystals through cation-exchange-mediated nucleation. Angew. Chem. Int. Ed. 47, 3029-3033 (2008).

14. Jain, P. K., Amirov, L., Aloni, S. \& Alivisatos, A. P. Nanoheterostructure cation exchange: anionic framework preservation. J. Am. Chem. Soc. 132, 9997-9999 (2010).

15. Li, J., Zhang, T., Yin, Y. \& Zhong, W. Fluorescence signal amplification by cation exchange in ionic nanocrystals. Angew. Chem. Int. Ed. 48, 1588-1591 (2009).

16. Sahu, A. et al. Electronic impurity doping in CdSe nanocrystals. Nano Lett. 12, 2587-2594 (2012).

17. Mocatta, D. et al. Heavily doped semiconductor nanocrystal quantum dots. Science 332, 77-81 (2011).

18. Norris, D. J., Sacra, A., Murray, C. B. \& Bawendi, M. G. Measurement of the size dependent hole spectrum in CdSe quantum dots. Phys. Rev. Lett. 72, 2612-2615 (1994).

19. Opik, A. I. \& Varvas, J. A. Defect structure of Cu-Doped cadmium selenide. Phys. Stat. Sol. 74, 467-473 (1982).

20. Grandhi, G. K., Tomar, R. \& Viswanatha, R. Study of surface and bulk electronic structure of II-VI semiconductor nanocrystals using $\mathrm{Cu}$ as a nanosensor. ACS Nano 6, 9751-9763 (2012).
21. Meulenberg, R. W. Structure and composition of Cu-doped CdSe nanocrystals using soft x-ray absorption spectroscopy. Nano Lett. 4, 2277-2285 (2004).

22. Sakalas, A. \& Baubinas, R. Scattering centers and their relation to the recombination centres in single crystals of CdSe. Phys. Stat. Sol. 31, 301-307 (1975).

23. Türe, I. E., Claybourn, M., Brinkman, A. ,W. \& Woods, J. Defects in cadmium selenide. J. Cryst. Growth 72, 189-193 (1985).

24. Kokubun, Y., Watanabe, H. \& Wada, M. Photoluminescence of CdSe single crystals. Jpn J. Appl. Phys. 13, 1393-1398 (1974).

25. Monod, J., Wyman, J. \& Changeaux, J. P. On the nature of allosteric transitions a plausible model. J. Mol. Biol. 12, 88-118 (1965).

26. Weiss, J. N. The Hill equation revisited: uses and misuses. FASEB J. 11, 835-841 (1997).

27. Hainovsky, N. \& Maier, J. Premelting, sublattice melting, and overall melting in Frenkel disordered ionic crystals. Solid State Ionics 76, 199-205 (1995).

28. Fayet, P. et al. The role of small silver clusters in photography. Z. Phys. D 3, 299-302 (1986).

29. Gurney, R. W. \& Mott, N. F. The theory of the photolysis of silver bromide and the photographic latent image. Proc. Roy. Soc. 164, 151-167 (1937).

30. Mott, N. F. The photographic process. Nature 175, 234-236 (1955).

31. Rivest, J. B. \& Jain, P. K. Cation exchange on the nanoscale: an emerging technique for new material synthesis, device fabrication, and chemical sensing. Chem. Soc. Rev. 42, 89-96 (2013).

32. Baumgartner, J. et al. Nucleation and growth of magnetite from solution. Nat Mater. 12, 310-314 (2012)

33. Gebauer, D., Völkel, A. \& Cölfen, H. Stable prenucleation calcium carbonate clusters. Science 322, 1819-1822 (2008).

34. Carbone, L. et al. Synthesis and micrometer-scale assembly of colloidal CdSe/ CdS nanorods prepared by a seeded growth approach. Nano Lett. 7, 2942-2950 (2007).

35. Yu, W. W., Qu, L., Guo, W. \& Peng, X. Experimental determination of the extinction coefficient of CdTe, CdSe, and CdS nanocrystals. Chem. Mater. 15, 2854-2860 (2003).

\section{Acknowledgements}

We thank Melika Fini and Alex Stanton for assistance with preliminary experiments. P.K.J. acknowledges support from the DuPont Young Professor Award and Frederick Seitz Materials Research Laboratory, where characterization was performed.

\section{Author contributions}

S.L.W. prepared samples, designed and performed measurements, analysed and interpreted data and co-wrote the manuscript. J.G.S. performed electron microscopy characterization. M.B. performed XPS measurements. P.K.J. conceived project, designed experiments, developed concepts and theory, analysed and interpreted data and wrote the manuscript.

\section{Additional information}

Supplementary Information accompanies this paper at http://www.nature.com/ naturecommunications

Competing financial interests: The authors declare no competing financial interests.

Reprints and permission information is available online at http://npg.nature.com/ reprintsandpermissions/

How to cite this article: White, S.L. et al. Co-operativity in a nanocrystalline solid-state transition. Nat. Commun. 4:2933 doi: 10.1038/ncomms3933 (2013). 